\title{
Long-standing insulinoma: two case reports and review of the literature
}

\author{
Mohamed Tarchouli ${ }^{1 *}$, Abdelmounaim Ait Ali ${ }^{1}$, Moulay Brahim Ratbi ${ }^{1}$, Mohamed said Belhamidi ${ }^{1}$, \\ Mohamed Essarghini ${ }^{1}$, El Mehdi Aboulfeth' ${ }^{1}$, Mohamed Bouzroud ${ }^{1}$, Yassir Sbitti ${ }^{2}$, Mohamed Oukabli ${ }^{3}$, \\ Mohammed Elfahssi ${ }^{1}$ and Khalid Sair ${ }^{1}$
}

\begin{abstract}
Background: Insulinomas are rare pancreatic endocrine tumors. Most are benign and solitary. However, the nonspecific symptoms and small size of these tumors led to difficulties of diagnosis and localization.

Case presentation: We present two Arab patients with pancreatic long-standing insulinoma. Both patients presented episodic hypoglycemic symptoms respectively during 10 and 2 years. Biochemical and morphological workup detected localized pancreatic insulinoma. Open procedure surgery was done for the two patients and insulinomas were successfully removed by enucleation.

Conclusion: Insulinoma remains a diagnostic challenge to practitioners. Diagnosis of suspected cases is easily confirmed by standard endocrine tests, especially the supervised fasting test. Accurate preoperative localization is essential for more effective and safest surgery.
\end{abstract}

Keywords: Insulinoma, Pancreas, Diagnosis, Management, Surgery

\section{Background}

Among pancreatic endocrine tumors, insulinoma is the most common type. This tumor was reported in 1-4 people per one million person years [1]. It can be seen at any age and occurs slightly more frequently in female than male [2]. Usually insulinoma clinical presentation is benign, with a solitary and small size $(<2 \mathrm{~cm}$ in diameter). Most are sporadic, however, $10 \%$ of insulinomas are multiple and occur as part of multiple endocrine neoplasia syndrome type I (MEN-I). Because of nonspecific symptoms, insulinoma may be misdiagnosed with other disorders. Patients often present with hypoglycemia signs resulting from inappropriate insulin secretion [3]. After biochemical confirmation of hyperinsulinism, preoperative localization of the tumor in the pancreas may be difficult. Surgical removal, often curative, continues to be the treatment of choice.

\footnotetext{
*Correspondence: mtarchouli@gmail.com

${ }^{1}$ Department of Digestive Surgery, Faculty of Medicine and Pharmacy, Mohammed V Military Hospital, Mohammed V University, Rabat, Morocco Full list of author information is available at the end of the article
}

In this manuscript, we report 2 cases of pancreatic insulinoma, and we discuss diagnosis, localization and management of this uncommon disease.

\section{Case presentation 1}

A 60-year-old Arab male had history of episodic and repetitive symptoms including diaphoresis, tremors, palpitations, and occasional loss of consciousness since 10 years. These symptoms, associated with a weight gain and a chronic weakness, occurred especially at night away from meals and were relieved with eating something. He had no family history of endocrine disease. Physical examination showed a healthy man with a Body Mass Index (BMI) of $34.9 \mathrm{~kg} / \mathrm{m}^{2}$. Blood laboratory tests demonstrated a low initial glucose level at $46 \mathrm{mg} / \mathrm{dl}$ $(70-11 \mathrm{mg} / \mathrm{dl})$, a high plasma insulin level at $70.4 \mu \mathrm{IU} / \mathrm{ml}$ $(2.6-24.9 \mu \mathrm{IU} / \mathrm{ml})$, and a high C-peptide level at $6.76 \mathrm{ng} /$ $\mathrm{ml}(0.8-4.2 \mathrm{ng} / \mathrm{ml})$. Prolonged supervised fasting test was applied and produced symptomatic hypoglycemia with hyperinsulinemia. Urine for sulfonylurea screen was negative. Abdominal computed tomography (CT) scan with contrast demonstrated a well-defined hypervascular 
lesion involving uncinate process of pancreas measuring $15 \mathrm{~mm}$ in diameter with enhancement during the arterial phases of contrast bolus (Fig. 1), but without liver metastasis or intra-abdominal lymph nodes. Magnetic resonance imaging (MRI) of brain was also performed showing an enlarged pituitary gland without nodular lesions. Other hormonal studies including serum cortisol level, parathormone level, adrenocorticotropic hormone (ACTH) level, and thyroid function were normal. Regarding the normal hormonal assessment, multiple endocrine neoplasia was eliminated.

Open surgical exploration was made. Complete mobilization and careful bimanual palpation of the pancreas allowed identifying a firm and well-circumscribed nodule at the uncinate process. Nodule enucleation was performed and pathological examination revealed an encapsulated pancreatic mass measuring $15 \times 15 \mathrm{~mm}$. Mitotic index was about 4 per 10 high-power fields (HPF) and proliferation index ki-67 was estimated at $10 \%$. Immunohistochemically, tumor cells showed a positive and diffuse staining for synaptophysin. Complementary staining for insulin was not preformed because not available in our institution. Therefore, an intermediate grade neuroendocrine tumor of pancreas was identified. Regarding to functional status and biological behavior, diagnosis of pancreatic insulinoma was confirmed. Immediately after surgical treatment, the glucose level increased to the normal range. The patient was discharged without any hypoglycemic symptoms after 4 days. The patient remains asymptomatic within 6 months follow up.

\section{Case presentation 2}

A 47-year-old Arab female suffered from hypoglycemic attacks characterized by dizziness, fatigue,

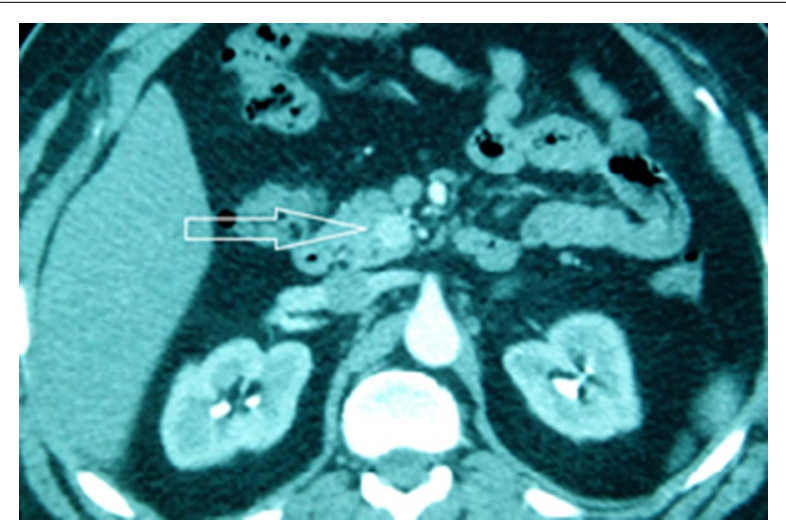

Fig. 1 Axial computed tomography scan image: mass in the uncinate process of pancreas measuring $15 \mathrm{~mm}$ in diameter with bright contrast enhancement during arterial phase (arrow), without pathologic intra- abdominal lymph nodes tremulousness, sweating, and hunger, associated with weight gain for 2 years. Most episodes occurred in the evening with a worsening of symptoms during prolonged fasting of Ramadan. She had no family history of endocrine disease. Physical examination showed a well-nourished patient with a BMI of $52.5 \mathrm{~kg} / \mathrm{m}^{2}$. Blood laboratory tests revealed, a low glucose level at $39 \mathrm{mg} / \mathrm{dl}$ (70-11 mg/dl); a high plasma insulin level at $65.2 \mu \mathrm{IU} /$ $\mathrm{ml}(2.6-24.9 \mu \mathrm{IU} / \mathrm{ml})$, and a C-peptide level at $4.99 \mathrm{ng} /$ $\mathrm{ml}(0.8-4.2 \mathrm{ng} / \mathrm{ml})$. Prolonged supervised fasting test was applied and produced symptomatic hypoglycemia with hyperinsulinemia. Urine for sulfonylurea screen was negative. Abdominal CT scan with contrast demonstrated a hypervascular lesion involving head of pancreas measuring $20 \times 17 \mathrm{~mm}$ with enhancement during the arterial phases of contrast bolus, but without liver metastasis or intra-abdominal lymph nodes. Abdominal MRI showed a nodule located at the junction head uncinate process of pancreas with intimate relationship to the second portion of duodenum but without bile or pancreatic ducts dilatation (Fig. 2). Thus, endoscopic ultrasonography (EUS) was performed showing a rounded hypoechoic mass of pancreatic head. MRI of hypophysis and cervical
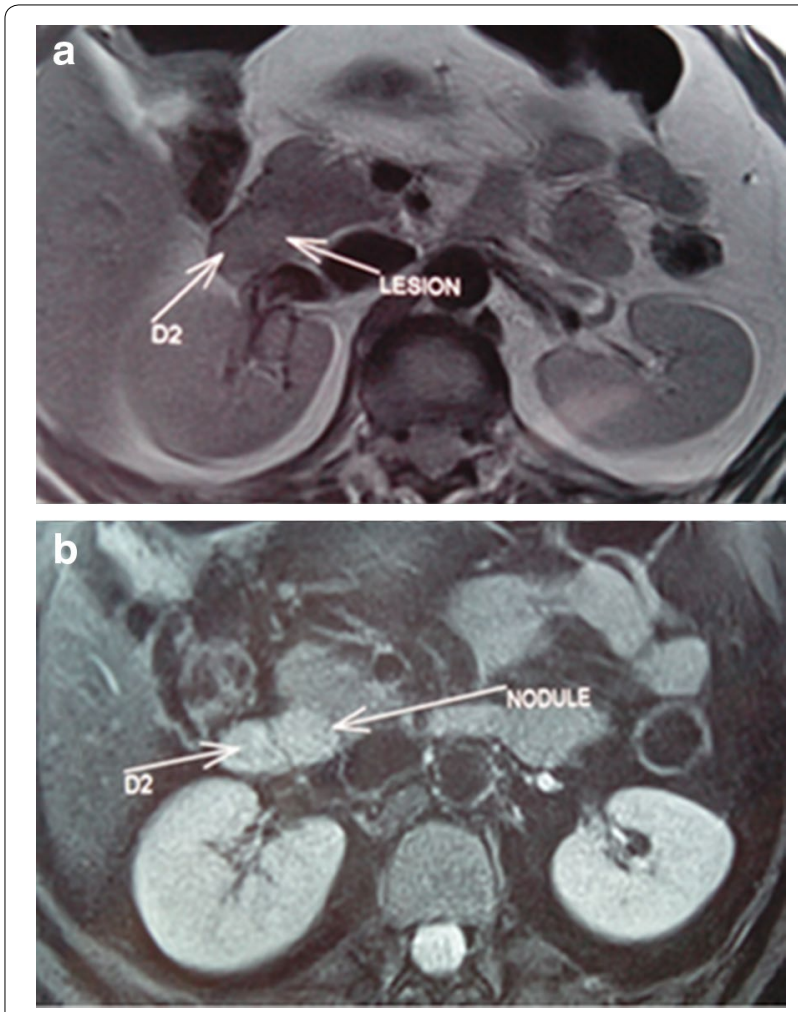

Fig. 2 Abdominal magnetic resonance imaging findings: pancreatic nodule with low signal intensity on T1-weighted images (lesion arrow) (a) and high signal intensity on T2-weighted images (nodule arrow) (b) 
ultrasound were normal. Other hormonal studies including serum cortisol level, parathormone level, ACTH level and thyroid function were normal eliminating a multiple endocrine neoplasia.

Open surgical exploration was made. Complete mobilization and careful bimanual palpation of the pancreas discovered a firm and unifocal nodule in the pancreatic head without relationship to the bile duct. Nodule enucleation was achieved (Fig. 3) and pathological evaluation has revealed an encapsulated pancreatic mass measuring $20 \times 15 \mathrm{~mm}$ (Fig. 4). Mitotic index was about 2 per $10 \mathrm{HPF}$ and proliferation index ki-67 was estimated at $10 \%$. Immunohistochemically, tumor cells showed a positive and diffuse staining for synaptophysin (Fig. 5).

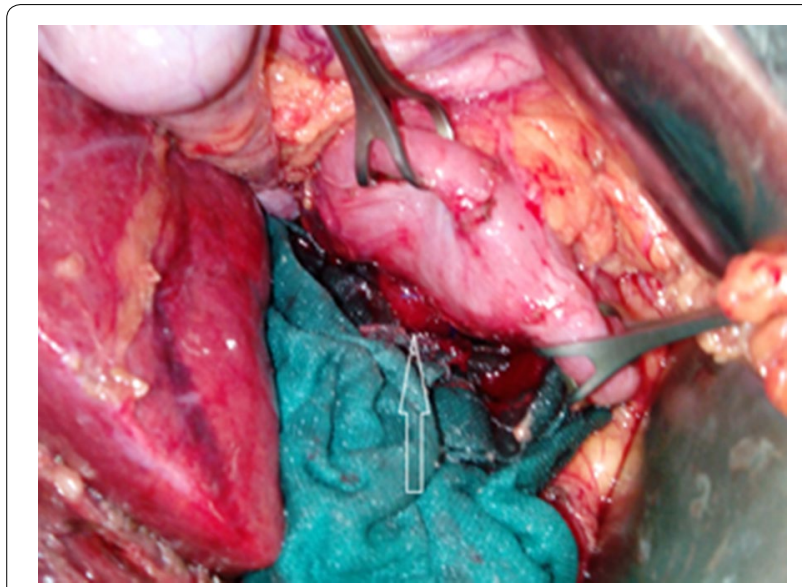

Fig. 3 Operative view showing a nodule in the pancreatic head with intimate relationship to the second portion of duodenum (arrow)
Complementary staining for insulin was not preformed because not available in our institution. Finally, an intermediate grade neuroendocrine tumor of pancreas was identified. Regarding to functional status and biological behavior, diagnosis of pancreatic insulinoma was confirmed. Immediately after surgical treatment, the glucose level increased to the normal range. The patient was discharged without any hypoglycemic symptoms after 5 days. The patient is currently in 1 year follow-up with a good evolution.

\section{Discussion}

Sporadic distribution, small size and high benignity rate are known insulinoma features, however etiopathogenesis remains still unclear. This rare tumor may have variable and nonspecific presentations all referable to the hypoglycemic state. Hypoglycemic symptoms can be divided into neuroglycopenic signs, most common, including confusion, behavioral changes, visual disturbances, weakness, dizziness, seizures and loss of consciousness, and neurogenic signs, such as anxiety, sweating, palpitations, tremors and feeling of warmth $[4$, 5].These symptoms become typically evident after fasting and are often precipitated by physical exercises. However, the median duration of symptoms before diagnosis remains variable and can reach $12-18$ months on average or even years in rare cases [2]. In our report, insulinoma diagnosis was delayed for several years. Simply because symptoms of hypoglycemia have been misinterpreted and misattributed to cardiac and neurological disorders before the insulinoma was recognized. In addition, our two patients tried to avoid hypoglycemic signs by eating frequently with resultant weight gain.
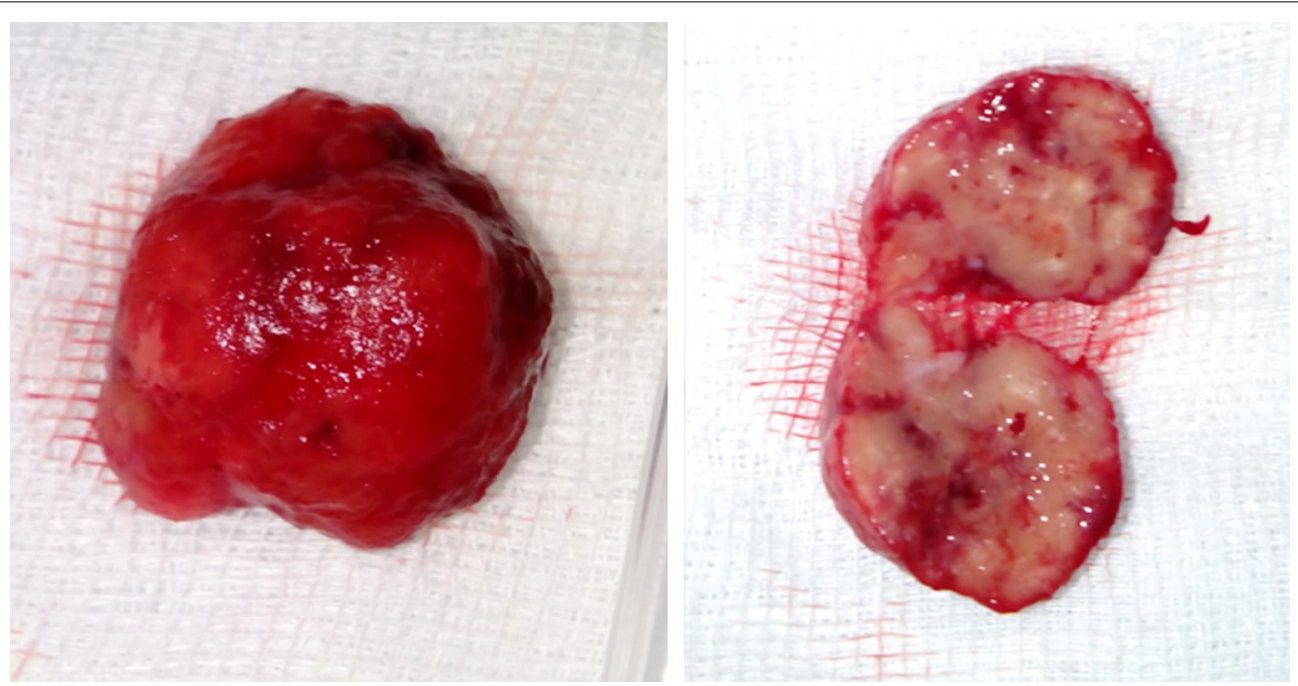

Fig. 4 Resected specimen 

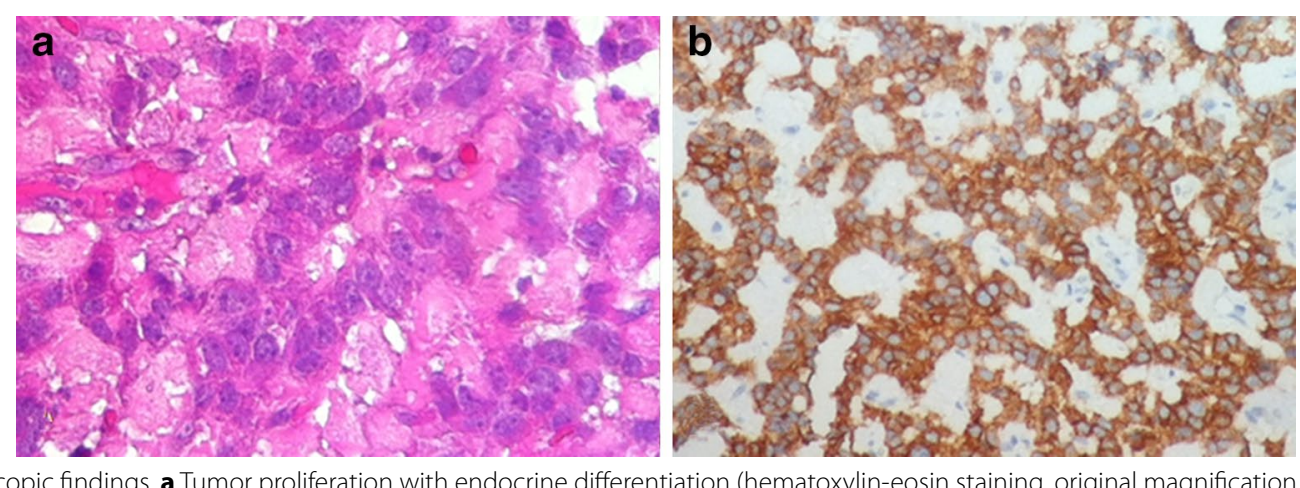

Fig. 5 Microscopic findings. a Tumor proliferation with endocrine differentiation (hematoxylin-eosin staining, original magnification $\times 40$ ). $\mathbf{b}$ Strong and diffuse expression of synaptophysin (immunohistochemistry, original magnification $\times 100$ )

Insulinoma is still suggested by the Whipple's triad including: symptoms of hypoglycemia induced by fasting or exercise, plasma glucose level below than $45 \mathrm{mg} / \mathrm{dl}$ and relief of symptoms following the administration of glucose. The supervised 72-h fasting test remains to be the gold standard for biochemical diagnosis with measurement of plasma glucose, insulin, C-peptide, and proinsulin during the onset of hypoglycemic symptoms. We used this test in our two cases to diagnose hyperinsulinism.

Various preoperative procedures can be used to localize the tumor in order to plan therapeutic strategy. The choice of procedure depends upon which tests are available and local radiologic skills. In our context, multiple noninvasive and invasive options are used including trans-abdominal ultrasonography, CT scan, MRI and EUS. The reported sensitivity of conventional CT and MRI for detection of pancreatic insulinoma ranges respectively from 33 to 64 and 40 to $90 \%$. However, the advent of helical CT scan has enabled detection of about $94 \%$ of insulinomas $[1,4]$. Consequently, it is currently accepted that $\mathrm{CT}$ scan is the first-line and MRI is the second-line investigation. These modalities can identify the exact size and location of an insulinoma, describe its anatomic relationship to surrounding structures and detect the presence of metastatic lesions suggestive of malignancy [6].

Some authors consider EUS as the best exam for preoperative localization of insulinoma, with a sensitivity of up to $94 \%$. It can detect even small tumors of $5 \mathrm{~mm}$, and reveal important relation to the bile duct and adjacent blood vessels. Also, EUS allows performing fine-needle aspiration cytology of suspicious lesions and preoperative marking of tumors to facilitate surgical excision particularly with laparoscopic approach. However, EUS findings depend largely on the examiner's experience $[1,2]$. In our institution, we generally use this technique when the tumor is not detected with the previously mentioned imaging modalities.
Bimanual palpation combined with intraoperative ultrasonography (IOUS) is the most effective method to detect more than $95 \%$ of tumors, but requires complete mobilization of the pancreas [7, 8]. Because of this operative success particularly in the hands of experienced surgeons, some authors suggest that preoperative localization studies are not necessary $[9,10]$. However, with recent advances in imaging techniques, preoperative topographic assessment is considered useful in avoiding blind resection and planning for rapid, accurate and safe surgery. Additionally, there is absolutely no question that positive localization is required prior to re-operative insulinoma [4].

Most insulinomas can be cured with surgery. Surgical procedure choice depends on the size and location of the mass. Tumor enucleation is the procedure of choice especially in case of small and solitary nodule that is not encroaching on the pancreatic or bile ducts [11]. The lesions are typically reddish-brown, firm, and encapsulated with a clear plane of dissection between the tumor and surrounding soft pancreatic parenchyma [12]. In addition, recent guidelines suggest that enucleation is enough in front of a well-circumscribed lesion, clearly localized before surgery, near or at the pancreatic surface, and easily defined intra-operatively [4]. Moreover, pancreatic resection is indicated for lesions invading or in close proximity to the pancreatic duct or major vessels, or suspicious for malignancy with a hard, infiltrating tumor and puckering of the surrounding soft tissue, pancreatic duct dilatation or lymph node involvement $[4,13]$. Resection options include distal pancreatectomy (with or without splenectomy), Whipple procedure (pancreaticoduodenectomy), or median pancreatectomy, depending on the site of insulinoma.

If the tumor is not identified despite a careful surgical exploration with bimanual palpation and IOUS, termination of the surgical procedure without blind resection 
is recommended. In such cases, the patient should be evaluated and re-operated at a referral center. Blind distal pancreatectomy is currently not appropriate and must be avoided [10, 14]. Consequently, more extensive localization procedures must be applied before reoperation, often including the intra-arterial calcium stimulation test with hepatic venous sampling (IACS-test). This test helps to regionalize the lesion preoperatively with a high detection rate ranging from 94 to $100 \%$. [15]. Therefore, the use of IACS-test allows for a more accurate surgical approach and can minimize the likelihood of reoperation. It may be appropriate when an insulinoma is strongly suspected but all previously described tests are negative. However, high cost and exclusive availability in some specialized centers lead to reserve this modality for patients with persistent or recurrent hyperinsulinism after initial surgery [16].

Furthermore, laparoscopic approach is currently feasible and becomes increasingly reported with good results in selected patients $[17,18]$. Tumor location should be confirmed intra-operatively by laparoscopic ultrasonography $[19,20]$. Both of our patients underwent tumor enucleation using open surgical approach. The lesions were easily identified thanks to only bimanual palpation of the pancreatic parenchyma.

Histologically, insulinomas are epithelial neoplasms associated with strong and diffuse immunohistochemical expression of neuroendocrine markers such as synaptophysin and chromogranin. Mitotic rate (number of mitoses per $10 \mathrm{HPF}$ ) and proliferation index (Ki-67 labeling index) are particularly helpful to separate welldifferentiated from poorly differentiated tumors [21]. Conversely, Malignant insulinomas are difficult to distinguish histologically and often the diagnosis of malignancy is only made when metastases occur [10].

Medical management of insulinoma, used to treat and prevent hypoglycemia, is generally restricted to unresectable metastatic tumors, unsuccessful operation with persistent symptoms, inoperable patients, and patients awaiting or refusing surgery $[1,4]$. Moreover, other recent techniques for the management of insulinoma have been reported, including injection of octreotide, EUS guided alcohol ablation, radiofrequency ablation, or embolization of an insulinoma [1].

\section{Conclusion}

Insulinoma is a rare neuroendocrine tumor, usually benign, but can be life-threatening in causing hypoglycemic accidents. Biochemical diagnosis is easy, but preoperative localization may prove difficult. However, most insulinomas can be identified intraoperatively by experienced surgeons. Surgical resection remains the treatment of choice with an extremely high success rate.
Laparoscopic approach is increasingly performed and blind pancreatic resection is not recommended. Finally, medical options are reserved for unresectable or metastatic tumors.

\section{Consent}

Written informed consent was obtained from both patients for publication of this Case Report and any accompanying images. A copy of the written consent is available for review by the Editor-in-Chief of this journal.

\section{Abbreviations}

MEN-I: multiple endocrine neoplasia syndrome type I; BMI: Body Mass Index; ACTH: adrenocorticotropic hormone; CT: computed tomography; MRI: magnetic resonance imaging; EUS: endoscopic ultrasonography; HPF: high-power fields; IOUS: intraoperative ultrasonography; IACS-test: intra-arterial calcium stimulation test with hepatic venous sampling.

\section{Authors' contributions}

MT participated in the conception and design of the report and wrote the paper. MBR, MSB, MES, MA, MB, MEL and MO have made substantial contributions to acquisition, analysis and interpretation of patient's data. AAA and YS have been involved in coordination and design of the report and revision of the manuscript. KS participated in drafting and revision of the manuscript. All authors read and approved the final manuscript.

\section{Author details}

${ }^{1}$ Department of Digestive Surgery, Faculty of Medicine and Pharmacy, Mohammed V Military Hospital, Mohammed V University, Rabat, Morocco. ${ }^{2}$ Department of Oncology, Faculty of Medicine and Pharmacy, Mohammed V Military Hospital, Mohammed V University, Rabat, Morocco. ${ }^{3}$ Department of Pathology, Faculty of Medicine and Pharmacy, Mohammed V Military Hospital, Mohammed V University, Rabat, Morocco.

\section{Acknowledgements}

The authors would like to thank the team of Department of Digestive Surgery for providing support and helping in preparing this manuscript. Neither author received any source of funding for this manuscript.

\section{Compliance with ethical guidelines}

\section{Competing interests}

The authors declare that they have no competing interests.

Received: 3 April 2015 Accepted: 7 September 2015

Published online: 15 September 2015

\section{References}

1. Okabayashi T, Shima Y, Sumiyoshi T, Kozuki A, Ito S, Ogawa Y, et al. Diagnosis and management of insulinoma. World J Gastroenterol. 2013;19:829-37.

2. Bouslama K, Maghrebi H, Bedioui H, Bouslama K. Pancreatic insulinoma: diagnostic approach and therapeutic modalities. J Afr Hépatol Gastroentérol. 2014;8:11-5.

3. Patel S, Narwari M, Parekh D, Shah V. Insulinoma: case report and review of diagnostic and treatment modalities. J Assoc Physicians India. 2013;61:423-6.

4. Abboud B, Boujaoude J. Occult sporadic insulinoma: localization and surgical strategy. World J Gastroenterol. 2008;14:657-65.

5. Cryer PE. Symptoms of hypoglycemia, thresholds for their occurrence, and hypoglycemia unawareness. Endocrinol Metab Clin North Am. 1999;28:495-500, v-vi. 
6. McAuley G, Delaney H, Colville J, Lyburn I, Worsley D, Govender P, et al Multimodality preoperative imaging of pancreatic insulinomas. Clin Radiol. 2005;60:1039-50.

7. Correnti S, Liverani A, Antonini G, Paganelli MT, Mercati U. Intraoperative ultrasonography for pancreatic insulinomas. Hepatogastroenterology. 1996:43:207-11.

8. Norton JA. Intraoperative methods to stage and localize pancreatic and duodenal tumors. Ann Oncol. 1999;10(Suppl 4):182-4.

9. Hashimoto LA, Walsh RM. Preoperative localization of insulinomas is not necessary. J Am Coll Surg. 1999;189:368-73.

10. Shin JJ, Gorden P, Libutti SK. Insulinoma: pathophysiology, localization and management. Future Oncol. 2010;6:229-37.

11. Finlayson $\mathrm{E}_{,} \mathrm{Clark} \mathrm{OH}$. Surgical treatment of insulinomas. Surg Clin North Am. 2004;84:775-85.

12. Vanderveen K, Grant C. Insulinoma. Cancer Treat Res. 2010;153:235-52.

13. Grant CS. Insulinoma. Best Pract Res Clin Gastroenterol. 2005;19:783-98.

14. Hirshberg B, Libutti SK, Alexander HR, Bartlett DL, Cochran C, Livi A, et al. Blind distal pancreatectomy for occult insulinoma, an inadvisable procedure. J Am Coll Surg. 2002;194:761-4.
15. Morita S, Machida H, Kuwatsuru R, Saito N, Suzuki K, lihara M, et al. Preoperative localization of pancreatic insulinoma by super selective arterial stimulation with venous sampling. Abdom Imaging. 2007;32:126-8.

16. Tseng LM, Chen JY, Won JG, Tseng HS, Yang AH, Wang SE, et al. The role of intra-arterial calcium stimulation test with hepatic venous sampling (IACS) in the management of occult insulinomas. Ann Surg Oncol. 2007:14:2121-7.

17. Isla A, Arbuckle JD, Kekis PB, Lim A, Jackson JE, Todd JF, et al. Laparoscopic management of insulinomas. Br J Surg. 2009;96:185-90.

18. Jaroszewski DE, Schlinkert RT, Thompson GB, Schlinkert DK. Laparoscopic localization and resection of insulinomas. Arch Surg. 2004;139:270-4.

19. lihara M, Kanbe M, Okamoto T, Ito Y, Obara T. Laparoscopic ultrasonography for resection of insulinomas. Surgery. 2001;130:1086-91.

20. Lo CY, Lo CM, Fan ST. Role of laparoscopic ultrasonography in intraoperative localization of pancreatic insulinoma. Surg Endosc. 2000;14:1131-5.

21. Klimstra DS, Modlin IR, Coppola D, Lloyd RV, Suster S. The pathologic classification of neuroendocrine tumors: a review of nomenclature, grading, and staging systems. Pancreas. 2010;39:707-12.

\section{Submit your next manuscript to BioMed Central and take full advantage of:}

- Convenient online submission

- Thorough peer review

- No space constraints or color figure charges

- Immediate publication on acceptance

- Inclusion in PubMed, CAS, Scopus and Google Scholar

- Research which is freely available for redistribution

Submit your manuscript at www.biomedcentral.com/submit
C Biomed Central 